\title{
Characterization of High-Dimensional Entangled Systems via Mutually Unbiased Measurements
}

\author{
D. Giovannini, ${ }^{1, *}$ J. Romero, ${ }^{1,2}$ J. Leach, ${ }^{3}$ A. Dudley, ${ }^{4}$ A. Forbes, ${ }^{4,5}$ and M. J. Padgett ${ }^{1}$ \\ ${ }^{1}$ School of Physics and Astronomy, SUPA, University of Glasgow, Glasgow G12 8QQ, United Kingdom \\ ${ }^{2}$ Department of Physics, SUPA, University of Strathclyde, Glasgow G4 ONG, United Kingdom \\ ${ }^{3}$ School of Engineering and Physical Sciences, SUPA, Heriot-Watt University, Edinburgh EH14 4AS, United Kingdom \\ ${ }^{4}$ CSIR National Laser Centre, PO Box 395, Pretoria 0001, South Africa \\ ${ }^{5}$ School of Physics, University of KwaZulu-Natal, Private Bag X54001, Durban 4000, South Africa
}

(Received 20 December 2012; published 2 April 2013)

\begin{abstract}
Mutually unbiased bases (MUBs) play a key role in many protocols in quantum science, such as quantum key distribution. However, defining MUBs for arbitrary high-dimensional systems is theoretically difficult, and measurements in such bases can be hard to implement. We show experimentally that efficient quantum state reconstruction of a high-dimensional multipartite quantum system can be performed by considering only the MUBs of the individual parts. The state spaces of the individual subsystems are always smaller than the state space of the composite system. Thus, the benefit of this method is that MUBs need to be defined for the small Hilbert spaces of the subsystems rather than for the large space of the overall system. This becomes especially relevant where the definition or measurement of MUBs for the overall system is challenging. We illustrate this approach by implementing measurements for a high-dimensional system consisting of two photons entangled in the orbital angular momentum degree of freedom, and we reconstruct the state of this system for dimensions of the individual photons from $d=2$ to 5 .
\end{abstract}

PACS numbers: 42.50.Tx, 03.65.Ud, 03.65.Wj, 03.67.Dd

Mutually unbiased bases (MUBs) [1,2] are a key concept in quantum science, as they are intimately related to the nature of quantum information [3-5]. Measurements made in one of a set of MUBs provide no information about the state if this was prepared in another basis from the same set. In quantum mechanics, the amount of information that can be extracted from a physical system is fundamentally limited by the uncertainty relations $[3,4]$. In this context, MUBs acquire a fundamental relevance because they serve as a test bed with which one can explore general uncertainty relations and, ultimately, complementarity [5]. Some important questions related to MUBs remain open [3,5]: what is the number of MUBs for an arbitrary dimension $d$, and why is mutual unbiasedness not enough to guarantee a strong uncertainty relation? While we do not seek to answer these questions, we provide an accessible experimental platform for exploring these problems by demonstrating measurements in complete sets of MUBs.

Many quantum information protocols depend upon the use of MUBs. For example, quantum key distribution (QKD) relies on the fact that measurements in one basis preclude knowledge of the state in any of the others [6-8]. In addition, MUBs play an important role in the reconstruction of quantum states $[1,9,10]$, where they have been

Published by the American Physical Society under the terms of the Creative Commons Attribution 3.0 License. Further distribution of this work must maintain attribution to the author(s) and the published article's title, journal citation, and DOI. successfully used to enable the optimal reconstruction of entangled states of polarization [11] and single-photon linear momentum states [12].

It is known that a Hilbert space of dimension $D$ will have at most $D+1$ MUBs $[1,2,13]$. In 1989, Wootters showed that if one can find $D+1$ mutually unbiased bases in dimension $D$, these bases provide a set of measurements that can be used to optimally determine the density matrix of a $D$-dimensional system [1]. However, this approach rapidly breaks down for large $D$ for two reasons: first, defining MUBs in high dimensions becomes increasingly difficult [5,14], and second, performing the measurements in a complete high-dimensional set of MUBs becomes experimentally challenging $[11,15]$. This is especially relevant for multilevel multiparticle systems, where the dimension of the overall system scales as $D=d^{N}$, with $d$ the dimension of the Hilbert spaces of the $N$ individual particles.

We show experimentally that the alternative approach of performing local measurements in the MUBs of the single particles of a multiparticle system still allows a complete reconstruction of the overall density matrix with a minimum number of measurements [16]. The significant benefit of our procedure is that it only requires the definition of MUBs in a Hilbert space of size $d=D^{1 / N}$; see Fig. 1. We illustrate this approach in the case of a photonic implementation of a bipartite multilevel entangled system $(d=\sqrt{D})$ using the orbital angular momentum of light.

In addition to the spin angular momentum, associated with polarization, light can also carry orbital angular 


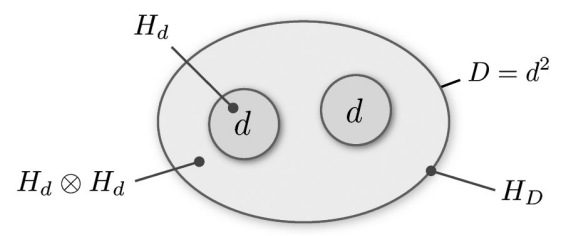

FIG. 1. Illustration of the state spaces of a bipartite system, where the system has dimension $D$ and each subsystem $d$. Adamson and Steinberg [11] performed measurements in the Hilbert space $H_{D}$ of the composite system, while we perform joint local measurements in the spaces $H_{d}$ of the individual subsystems (for $d=2-5$ ).

momentum (OAM) [17]. The OAM of light is associated with phase structures of the form $e^{i \ell \phi}$, where $\ell \hbar$ is the OAM carried by each photon and $\phi$ the azimuthal angle [18]. The unbounded Hilbert space of OAM is one example of a scalable high-dimensional resource that can be used for quantum information science [19-22]. For example, the entanglement of high-dimensional states provides implementations of QKD that are more tolerant to eavesdropping and can improve the bit rate in other quantum communication protocols [23-28].

One of the advantages of OAM is the ability to access $d$-dimensional subspaces [29], for each of which we can define all existing MUBs [30]. In this work, we implement measurements in high-dimensional MUBs within the OAM degree of freedom, and we show that the MUBs corresponding to $d$-dimensional subspaces are readily accessible with simple laboratory procedures. Furthermore, we show that measurements in MUBs of these subspaces can be used for the complete tomographic reconstruction of multipartite entangled systems with the minimum number of measurements. We produce entangled photon pairs by means of spontaneous parametric down-conversion that we then measure in full sets of $d+1$ MUBs for OAM, for dimensions ranging from $d=2$ to 5 . The states belonging to the MUBs are defined as superpositions of LaguerreGaussian modes.

Theory.-Consider two operators in a $d$-dimensional Hilbert space with orthonormal spectral decompositions. These operators, and their basis states, are said to be mutually unbiased $[1,2]$ if

$$
\left|\left\langle\psi_{m, i} \mid \psi_{n, j}\right\rangle\right|^{2}= \begin{cases}1 / d & \text { for } m \neq n \\ \delta_{i j} & m=n\end{cases}
$$

for all $i$ and $j$. The indices $i$ and $j$ correspond to the basis states, and $m$ and $n$ indicate any two bases. Operators that are quantum-mechanical observables are sometimes called mutually complementary, or maximally noncommutative [31]. This is because, given any eigenstate of one, the eigenvalue resulting from a measurement of the other is completely undetermined. In other words, the state of a system described in one MUB provides no information about the state in another. It is known that the number of
MUBs in dimension $d$ cannot exceed $d+1$ [1,5], and it is exactly $d+1$ if $d$ is prime or a prime power [1,32].

The simplest set of mutually unbiased observables can be found in dimension $d=2$. For example, in the twodimensional Hilbert space of polarization, the bases of horizontal (vertical), diagonal (antidiagonal), and left (right) circular polarizations provide a set of three MUBs. Two states belonging to the same basis are orthonormal, while the square of the inner product of any two states belonging to different bases is always $1 / 2$. Equivalent mutually unbiased states can be implemented using other two-dimensional state spaces, e.g., a subspace of OAM.

In our work, we choose to investigate the OAM degree of freedom of single photons. A general single-photon state in a $d$-dimensional subspace can be described by an orthonormal basis set of OAM modes $|\ell\rangle$ as $|\psi\rangle=\sum_{\{\ell\}} c_{\ell}|\ell\rangle$. The complex coefficients $c_{\ell}$ are subject to the normalization condition $\sum c_{\ell}^{2}=1$. Defining MUBs in a general $d$-dimensional space is a difficult problem [14]; however, for a number of low-dimensional cases, it is possible to find complete sets of MUBs using simple procedures [33]. For these cases, which include the dimensions $2-5$, the states $\{|\ell\rangle\}$ can be chosen to be one of the MUBs. The states belonging to the remaining $d$ MUBs are found to be superpositions of the basis states with coefficients of equal magnitude $\left|c_{\ell}\right|=1 / \sqrt{d}$ but differing phases.

In general, it is possible for a system to include more than one particle. If one considers a $d$-dimensional state space for each particle, the dimension $D$ of a system of $N$ particles will be $D=d^{N}$. Such a system will be unambiguously specified by its density matrix $\rho$, a positive-semidefinite unit-trace Hermitian operator that includes $d^{2 N}-1$ independent real parameters $\left(d^{4}-1\right.$ for a bipartite system).

MUBs play an important role in quantum state tomography $[1,34]$, the process of determining the density matrix of an unknown quantum system [35-37]. One approach to tomography is to perform measurements in the MUBs of the $D$-dimensional state space of the composite system [1]. However, such measurements are very challenging as they require the definition of MUBs for Hilbert spaces of very high dimension and can require the implementation of entangled observables [11]. Our approach is simpler as we use the MUBs of the state spaces of the single particles.

Let us consider for simplicity a bipartite system. An overcomplete set of measurements for the reconstruction of the $D$-dimensional system is provided by the pairwise combinations of all single-particle MUB states. The total number of independent measurements for this approach is equal to $[d(d+1)]^{2}$, which is always greater than $d^{4}-1$. We propose another suitable set of measurements, given by pairwise combinations of states from an appropriate subset of the overcomplete set. This subset contains all states in one MUB and all but one state in each of the remaining $d$ MUBs. It can be shown that the conditions for the completeness of a set of tomographic measurements [37] are satisfied by this reconstruction strategy [38]. 
This approach gives exactly the $d^{4}$ independent measurements that can then be used for a tomographically complete reconstruction of the $D$-dimensional system. The number of measurements in our method scales favorably with the dimension of the system if compared with other methods [38].

Experimental methods.-A 3 mm-thick $\beta$-barium borate (BBO) nonlinear crystal cut for type-I collinear spontaneous parametric down-conversion is pumped by a collimated $1 \mathrm{~W}$ UV laser to produce frequency-degenerate entangled photon pairs at $710 \mathrm{~nm}$. The copropagating signal and idler photons are separated by a nonpolarizing beam splitter and redirected to spatial light modulators (SLMs), onto which the output face of the crystal is imaged by a $2 \times$ telescope. In order for the crystal to produce twophoton states entangled over a wider range of OAM modes, we tune the phase-matching conditions of the BBO crystal to increase the OAM spectrum of the down-converted state [39]. The SLMs act as reconfigurable computer-generated holograms that allow us to measure any arbitrary superposition of OAM modes. The SLMs are used to modulate the phase and introduce a spatially dependent attenuation to discard light into the zero diffraction order, allowing the manipulation of the complex amplitude of the incoming light $[30,40,41]$.

We pump the crystal with a plane phase front. In order to observe correlations in all bases (instead of anticorrelations), the hologram displayed in one of the two detection arms is phase conjugate with respect to the other [19]. The projected Gaussian mode is then imaged onto a singlemode fiber that is coupled to a single-photon photodiode detector. The detectors' outputs are routed to coincidencecounting electronics with a timing window of $10 \mathrm{~ns}$. Narrow band, $10 \mathrm{~nm}$ interference filters are placed in front of the detectors to ensure that the frequency spread of the detected down-converted fields is small compared to the central frequencies.

The combination of the two SLMs, single-mode fibers, and coincidence-counting electronics allows us to perform projective measurements on the entangled state of photons $A$ and $B$ described by the operators

$$
\Pi_{m, i ; n, j}=\left|\psi_{m, i}\right\rangle_{A}\left|\psi_{n, j}\right\rangle_{B}^{*}\left\langle\psi _ { m , i } | _ { A } \left\langle\left.\psi_{n, j}\right|_{B} ^{*} .\right.\right.
$$

Here, the single-photon states $|\psi\rangle_{A}$ and $|\psi\rangle_{B}$ belong to MUBs in $d$ dimensions and are given by

$$
\left|\psi_{m, i}\right\rangle=\sum_{\{\ell\}} c_{m, i, \ell}|\ell\rangle
$$

where $c_{m, i, \ell}$ is a complex coefficient. The indices $m$ and $n$, which correspond to the basis indices, range from 1 to $d+1$; the indices $i$ and $j$, which represent a state within a basis, range from 1 to $d$. For each dimension $d$, we choose one set of OAM states $\{|\ell\rangle\}$. The OAM values used are $\{\ell\}=\{-2,+2\}$ for $d=2,\{-2,-1,+1,+2\}$ for $d=4$, and $\{-\lfloor d / 2\rfloor, \ldots,+\lfloor d / 2\rfloor\}$ for $d=3$ and 5. For each $d$, we take the basis corresponding to $m=1$ to be the

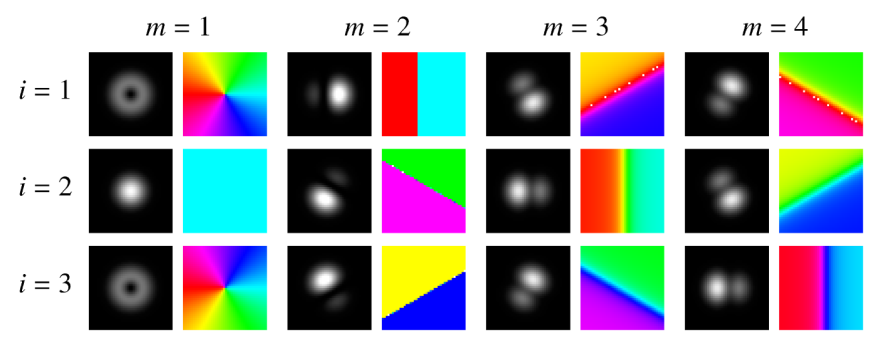

FIG. 2 (color online). Mutually unbiased modes $i$ for each of the 4 bases $m$ in $d=3$. The gray scale images represent the intensity, the color images represent the phase. The first basis, $m=1$, corresponds to Laguerre-Gaussian modes with OAM ranging from $\ell=-1$ to +1 .

orthonormal basis given above; the remaining bases are composed of superpositions of the $m=1$ states with appropriate complex coefficients; see Fig. 2. For the dimensions considered, the magnitude of these complex coefficients is $1 / \sqrt{d}$ for all $i$ and $\ell$.

To determine the phase terms $c_{m, i, \ell}$ that define the MUBs (for $m=2$ to $d+1$ ), we use the methods outlined by Refs. [33,42]. The coefficients are given by the mutually unbiased vectors derived from $d \times d$ dephased Hadamard matrices. These matrices are unique for $d=2,3,4$, and 5. For $d=2$, the MUBs obtained are the familiar set of bases that one usually associates with polarization states. Consequently, the two-dimensional MUBs for OAM [43] are the analogue of those for polarization [44]. All the modes used for $d=3$ are shown in Fig. 2.

An overcomplete set of measurements is obtained by scanning through all possible values of $m$ and $i$, for photon $A, n$, and $j$, for photon $B$. For every combination of $m, n, i$, and $j$, we record the coincidence counts and both the single-channel counts resulting from the projective measurement. From this set of data we extract the tomographically complete set of measurements previously described. These count rates are converted to detection probabilities through the following relationship:

$$
p_{k}=\frac{d^{2}}{\sum C_{k}} \frac{C_{k}-U_{k}}{U_{k}},
$$

where the index $k$ corresponds to a unique choice of measurement settings $m, n, i$, and $j, C_{k}$ is the coincidence count rate and $U_{k}$ is the anticipated uncorrelated coincidence rate, which is estimated by taking the product of the single-channel count rates and the gate time (Fig. 3). The normalization approach that we take accounts for different hologram efficiencies for different modes [38].

The task of the fitting procedure is to find the optimal density matrix $\rho$ of the $D$-dimensional system that best reproduces the experimental results. The parameters of the density matrix are established through numerical minimization of Pearson's cumulative test statistic $[45,46]$

$$
\chi^{2}=\sum_{k=1}^{d^{4}} \frac{\left(p_{k}-p_{k}^{\prime}\right)^{2}}{p_{k}^{\prime}}
$$


(a) $d=2\left(D=2^{2}\right)$

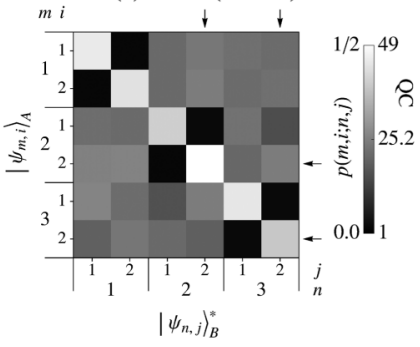

(c) $d=4\left(D=4^{2}\right)$
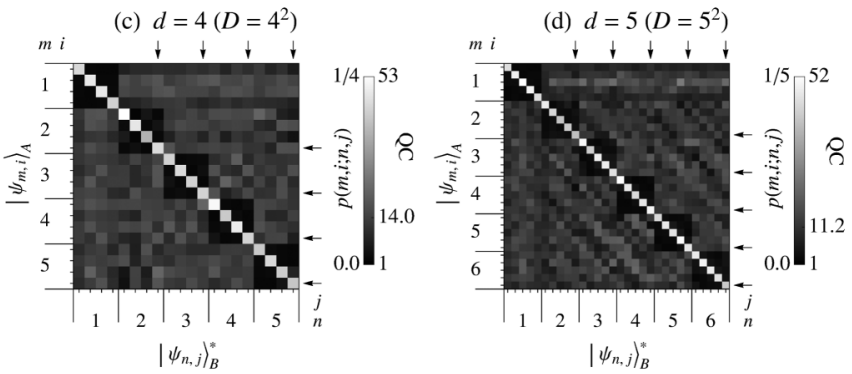

FIG. 3. Joint probabilities of detecting photon $A$ in state $\left|\psi_{m, i}\right\rangle_{A}$ and photon $B$ in state $\left|\psi_{n, j}\right\rangle_{B}$. The results are normalized such that the sum of the joint detection probabilities for measurements in any two bases $m$ and $n$ are unity. Therefore, the probabilities represented by the leading diagonal are expected to be $1 / d$, and all probabilities for $m \neq n$ are expected to be $1 / d^{2}$. We also display the quantum contrast (QC), which is given by the ratio of the measured coincidence rate to that of the expected accidental coincidences. The arrows indicate the rows and columns of measurements not required for the complete tomographic reconstruction of the density matrix.

where $p_{k}$ are the probabilities from the experiment, and $p_{k}^{\prime}=\operatorname{Tr}\left[\rho \Pi_{k}\right]$ are those predicted from the reconstructed density matrix.

The reconstructed density matrices for dimensions 2,3,4, and 5 are shown in Fig. 4. For each reconstructed density matrix $\rho$, we calculate the linear entropy $S=1-\operatorname{Tr}\left(\rho^{2}\right)$ and the fidelity $F=\operatorname{Tr}[\sqrt{\sqrt{\sigma} \rho \sqrt{\sigma}}]^{2}$, where $\sigma$ is the $D$-dimensional maximally entangled density matrix associated with arbitrarily large spiral bandwidth [39] and perfect detection. The uncertainties were calculated by repeating the reconstruction process for statistically equivalent copies of the original experimental data sets, each obtained by adding Poissonian fluctuations to the measured counts.

The reconstructed density matrices have low entropies, indicating pure states, and very high fidelities with respect to the maximally entangled state. Because of the finite spiral bandwidth of our generated state [39,47] and limitations in our measurement system, one would anticipate the fidelities to decrease and the entropies to increase as the dimension increases. Indeed, we observe this characteristic in our results.

For comparison, we also implemented the approach described in Ref. [48]. We find comparable entropies and fidelities whichever approach is used [38]. However, our method requires significantly fewer measurements. For (a) $d=2$

(b) $d=3$

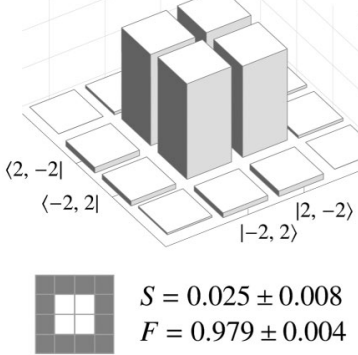

(c) $d=4$

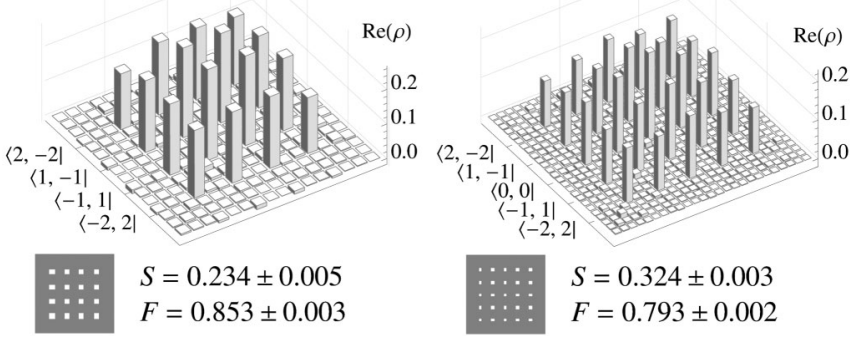

FIG. 4. Results of tomographic reconstructions using a complete set of single-photon mutually unbiased bases measurements. The real parts of the reconstructed density matrices $\rho$ are shown. Imaginary parts are less than 0.076 for $d=2,0.059$ for $d=3$, and 0.050 for $d=5,6$. Also shown are the linear entropy $S$ and fidelity $F$ for the reconstructed density matrices. Insets: Real parts of the theoretical density matrices for the maximally entangled states.

example, for $d=5$, the number of measurements required is $d^{4}=625$ compared to 2025 for the procedure outlined in Ref. [48]. Both methods rely on projective measurements in appropriate superpositions of the basis states in the dimension of choice. Neither is more experimentally demanding, as they can both be performed using the same setup and only differ in the choice of projection states.

The MUBs reconstruction method is applied here to almost maximally entangled states. The density matrices of maximally entangled states have low rank, $r<D$, and could thus be efficiently reconstructed through compressed sensing [49,50]. In the general case, however, a complete quantum state reconstruction by means of appropriately selected projection operators may be more appropriate and produce results with higher fidelity.

Conclusions.-In this work, we have demonstrated single-photon measurements for MUBs in the OAM degree of freedom and shown how these measurements can be used for efficient quantum state reconstruction. The procedure of measuring combinations of all single-photon states in one basis and all but one state in the remaining bases gives a minimal complete set of tomographic measurements. This experimental method can be readily applied to multilevel multipartite systems.

The OAM degree of freedom is becoming an important resource for quantum information science. Therefore, the ability to measure states in MUBs is an important step for quantum protocols implemented in this degree of freedom. 
Measuring MUBs in high-dimensional spaces is not just of practical importance for QKD protocols, but it can also provide important insight into the nature of information in physical systems.

We thank Adetunmise Dada and Daniel Tasca for useful discussions. This work was supported by EPSRC. We thank Hamamatsu for their support. M. J.P. thanks the Royal Society.

*d.giovannini.1@research.gla.ac.uk

[1] W. K. Wootters and B. D. Fields, Ann. Phys. (N.Y.) 191, 363 (1989).

[2] I. D. Ivanović, J. Phys. A 14, 3241 (1981).

[3] S. Wehner and A. Winter, New J. Phys. 12, 025009 (2010).

[4] S. M. Barnett, Quantum Information (Oxford University Press, Oxford, England, 2009).

[5] T. Durt, B.-G. Englert, I. Bengtsson, and K. Życzkowski, Int. J. Quantum. Inform. 08, 535 (2010).

[6] C. H. Bennett and G. Brassard, in Proceedings of the IEEE International Conference on Computers, Systems, and Signal Processing, Bangalore, 1984 (IEEE, Piscataway, NJ, 1984), p. 175.

[7] L. Jun-Lin and W. Chuan, Chin. Phys. Lett. 27, 110303 (2010).

[8] M. Malik, M. O’Sullivan, B. Rodenburg, M. Mirhosseini, J. Leach, M. P. J. Lavery, M. J. Padgett, and R. W. Boyd, Opt. Express 20, 13195 (2012).

[9] S. N. Filippov and V. I. Man'ko, Phys. Scr. T143, 014010 (2011).

[10] A. Fernández-Pérez, A. B. Klimov, and C. Saavedra, Phys. Rev. A 83, 052332 (2011).

[11] R. B. A. Adamson and A. M. Steinberg, Phys. Rev. Lett. 105, 030406 (2010).

[12] G. Lima, L. Neves, R. Guzmán, E. S. Gómez, W. A. T. Nogueira, A. Delgado, A. Vargas, and C. Saavedra, Opt. Express 19, 3542 (2011).

[13] S. Bandyopadhyay, P. O. Boykin, V. Roychowdhury, and F. Vatan, Algorithmica 34, 512 (2002).

[14] S. Brierley and S. Weigert, J. Phys. Conf. Ser. 254, 012008 (2010).

[15] E. Nagali, L. Sansoni, L. Marrucci, E. Santamato, and F. Sciarrino, Phys. Rev. A 81, 052317 (2010).

[16] R. T. Thew, K. Nemoto, A. G. White, and W. J. Munro, Phys. Rev. A 66, 012303 (2002).

[17] A. M. Yao and M. J. Padgett, Adv. Opt. Photonics 3, 161 (2011).

[18] L. Allen, M.W. Beijersbergen, R. J. C. Spreeuw, and J. P. Woerdman, Phys. Rev. A 45, 8185 (1992).

[19] A. Mair, V. Alipasha, G. Weihs, and A. Zeilinger, Nature (London) 412, 313 (2001).

[20] J. Leach, B. Jack, J. Romero, A. K. Jha, A. M. Yao, S. Franke-Arnold, D. G. Ireland, R. W. Boyd, S. M. Barnett, and M. J. Padgett, Science 329, 662 (2010).

[21] S. Gröblacher, T. Jennewein, A. Vaziri, G. Weihs, and A. Zeilinger, New J. Phys. 8, 75 (2006).

[22] V. D. Salakhutdinov, E. R. Eliel, and W. Löffler, Phys. Rev. Lett. 108, 173604 (2012).

[23] M. Bourennane, A. Karlsson, G. Björk, N. Gisin, and N. J. Cerf, J. Phys. A 35, 10065 (2002).
[24] N. J. Cerf, M. Bourennane, A. Karlsson, and N. Gisin, Phys. Rev. Lett. 88, 127902 (2002).

[25] H. Bechmann-Pasquinucci and W. Tittel, Phys. Rev. A 61, 062308 (2000).

[26] S. P. Walborn, D. S. Lemelle, M. P. Almeida, and P. H. Souto Ribeiro, Phys. Rev. Lett. 96, 090501 (2006).

[27] P. B. Dixon, G. A. Howland, J. Schneeloch, and J.C. Howell, Phys. Rev. Lett. 108, 143603 (2012).

[28] M. T. Gruneisen, J.P. Black, R.C. Dymale, and K.E. Stoltenberg, in Electro-Optical Remote Sensing, Photonic Technologies, and Applications VI, edited by G. W. Kamerman et al., SPIE Proceedings, Vol. 8542 (SPIE-International Society for Optical Engineering, Bellingham, WA, 2012), p. 85421Q.

[29] A. C. Dada, J. Leach, G. S. Buller, M. J. Padgett, and E. Andersson, Nat. Phys. 7, 677 (2011).

[30] M. T. Gruneisen, W. A. Miller, R. C. Dymale, and A. M. Sweiti, Appl. Opt. 47, A32 (2008).

[31] J. Schwinger, Proc. Natl. Acad. Sci. U.S.A. 46, 570 (1960).

[32] A. Klappenecker and M. Rötteler, Finite Fields and Applications (Springer, New York, 2004), Vol. 2948, pp. 262-266.

[33] S. Brierley, S. Weigert, and I. Bengtsson, Quantum Inf. Comput. 10, 0803 (2010).

[34] A. B. Klimov, C. Muñoz, A. Fernández, and C. Saavedra, Phys. Rev. A 77, 060303 (2008).

[35] D. F. V. James, P. G. Kwiat, W. J. Munro, and A. G. White, Phys. Rev. A 64, 052312 (2001).

[36] N. K. Langford, R. B. Dalton, M. D. Harvey, J. L. O'Brien, G. J. Pryde, A. Gilchrist, S. D. Bartlett, and A. G. White, Phys. Rev. Lett. 93, 053601 (2004).

[37] J. B. Altepeter, E. R. Jeffrey, and P. G. Kwiat, Advances in Atomic, Molecular, and Optical Physics (Elsevier, New York, 2005).

[38] See Supplemental Material at http://link.aps.org/ supplemental/10.1103/PhysRevLett.110.143601 for further details on the normalization of the experimental counts, the completeness of the tomographic method, the numerical reconstruction procedure, and a list of the MUBs employed.

[39] J. Romero, D. Giovannini, S. Franke-Arnold, S. M. Barnett, and M. J. Padgett, Phys. Rev. A 86, 012334 (2012).

[40] V. Arrizón, U. Ruiz, R. Carrada, and L. A. González, J. Opt. Soc. Am. A 24, 3500 (2007).

[41] J. A. Davis, D. M. Cottrell, J. Campos, M. J. Yzuel, and I. Moreno, Appl. Opt. 38, 5004 (1999).

[42] S. Brierley and S. Weigert, Phys. Rev. A 79, 052316 (2009).

[43] M. J. Padgett and J. Courtial, Opt. Lett. 24, 430 (1999).

[44] D. Bruß, Phys. Rev. Lett. 81, 3018 (1998).

[45] T. Opatrný, D.-G. Welsch, and W. Vogel, Phys. Rev. A 56, 1788 (1997).

[46] K. Banaszek, G. M. D’Ariano, M. G. A. Paris, and M. F. Sacchi, Phys. Rev. A 61, 010304 (1999).

[47] J. P. Torres, A. Alexandrescu, and L. Torner, Phys. Rev. A 68, 050301 (2003).

[48] M. Agnew, J. Leach, M. McLaren, F. S. Roux, and R. W. Boyd, Phys. Rev. A 84, 062101 (2011).

[49] D. Gross, Y.-K. Liu, S. T. Flammia, S. Becker, and J. Eisert, Phys. Rev. Lett. 105, 150401 (2010).

[50] W.-T. Liu, T. Zhang, J.-Y. Liu, P.-X. Chen, and J.-M. Yuan, Phys. Rev. Lett. 108, 170403 (2012). 